\title{
Interference of Temporally Distinguishable Photons Using Frequency-Resolved Detection
}

\author{
Venkata Vikram Orre, ${ }^{1,2}$ Elizabeth A. Goldschmidt, ${ }^{1,3}$ Abhinav Deshpande, ${ }^{1,4}$ Alexey V. Gorshkov, ${ }^{1,4}$ \\ Vincenzo Tamma, ${ }^{5,6}$ Mohammad Hafezi, ${ }^{1,2,7}$ and Sunil Mittal ${ }^{1,2, *}$ \\ ${ }^{1}$ Joint Quantum Institute, NIST/University of Maryland, College Park, Maryland 20742, USA \\ ${ }^{2}$ Department of Electrical and Computer Engineering and IREAP, University of Maryland, College Park, Maryland 20742, USA \\ ${ }^{3}$ U.S. Army Research Laboratory, Adelphi, Maryland 20783, USA \\ ${ }^{4}$ Joint Center for Quantum Information and Computer Science, NIST/University of Maryland, College Park, Maryland 20742, USA \\ ${ }^{5}$ School of Mathematics and Physics, University of Portsmouth, Portsmouth PO1 3QL, United Kingdom \\ ${ }^{6}$ Institute of Cosmology and Gravitation, University of Portsmouth, Portsmouth PO1 3FX, United Kingdom \\ ${ }^{7}$ Department of Physics, University of Maryland, College Park, Maryland 20742, USA
}

(Received 5 April 2019; published 17 September 2019)

\begin{abstract}
We demonstrate quantum interference of three photons that are distinguishable in time by resolving them in the conjugate parameter frequency. We show that the multiphoton interference pattern in our setup can be manipulated by tuning the relative delays between the photons, without the need for reconfiguring the optical network. Furthermore, we observe that the symmetries of our optical network and the spectral amplitude of the input photons are manifested in the interference pattern. We also demonstrate timereversed Hong-Ou-Mandel-like interference in the spectral correlations using time-bin entangled photon pairs. By adding a time-varying dispersion using a phase modulator, our setup can be used to realize dynamically reconfigurable and scalable boson sampling in the time domain as well as frequency-resolved multiboson correlation sampling.
\end{abstract}

DOI: 10.1103/PhysRevLett.123.123603

The nonclassical interference of two or more photons in an optical network is the fundamental phenomenon enabling many algorithms used in linear optics quantum computing [1-4], quantum communications [5-7], metrology [8,9], and boson sampling [10-12]. Quantum interference, such as Hong-Ou-Mandel (HOM) and Shih-Alley interference $[13,14]$, usually requires photons that are identical in their temporal and spectral degrees of freedom. Any distinguishability in the photons at the detectors leads to a reduction in the interference. The difficulty in experimentally generating identical photons has prompted strong interest in developing real world optical networks enabling the interference of nonidentical photons $[15,16]$. Recently, it was shown that nonclassical interference can be observed between photons completely distinguishable in time or frequency by exploiting correlation measurements in the corresponding conjugate parameter [17-21]. Remarkably, the interference can occur for any values of the input frequencies (or times) as long as the detector resolution in the conjugate parameter is sufficient to make the detectors "blind" to the spectral (or temporal) distinguishability of the photons. Furthermore, the temporal or spectral distinguishability can actually be used as a resource, for example, to reveal spectral properties of the input photons and the symmetries of the optical network $[19,20]$.

Many experiments have demonstrated interference of two photons that are distinguishable in frequency or time by resolving them in the conjugate parameter $[17,18,22,23]$.
Scaling these spectrally or temporally resolved interference phenomena to a larger number of photons can enable, for example, multiboson correlation sampling experiments where sampling over temporal or spectral modes, in addition to spatial modes, can relax the requirements on generating identical photons and could demonstrate quantum supremacy [24-27]. Indeed, time-resolved interference of three photons with different frequencies was demonstrated very recently where the temporal correlations between detected photons were manipulated using a spatial network of beam splitters [21]. In contrast, the complementary phenomenon, that is, frequency-resolved interference of multiple photons that are separated in time, allows for the convenient manipulations of spectral correlations by tuning the relative delays between photons, without reconfiguring the spatial network $[20,27]$. This scheme can operate in a single spatial (transverse) mode and, therefore, enable the realization of scalable temporal boson sampling using timevarying dispersion [28]. However, such frequency-resolved interference of more than two photons has not yet been demonstrated.

Here, we demonstrate frequency-resolved quantum interference of three photons that are completely distinguishable in time. We show that the interference observed in the spectral correlations of detected photons can be manipulated by changing the relative delays between the photons at the input. The interference is completely wiped out for longer delays between photons, that is when the spectral 
resolution of the detectors is not sufficient to erase the temporal distinguishability of the photons. Moreover, we observe that the symmetries of the optical network, and the spectral wave functions of photons are reflected in the measured spectral correlations. Finally, we also demonstrate spectral correlations in inverse-HOM interference using two time-bin entangled photons where both photons arrive either "early" or "late" [29]. In this case, we observe that the interference is sensitive to the phase between the two components of the entangled state, unlike the case of unentangled photons where interference is insensitive to small fluctuations in delay between the photons. Our experimental setup could easily be extended to introduce time-varying dispersion using phase modulators and realize the temporal boson sampling scheme of Ref. [28].

To demonstrate our scheme, we discuss first the interference of two temporally distinguishable photons in our setup (Fig. 1). The two-photon interference can be analyzed using the spectral correlation function $\Gamma\left(\omega_{1}, \omega_{2}, \tau\right)$, which is the probability of detecting two photons at the two detectors, with frequencies $\omega_{1}$ and $\omega_{2}$, respectively, and $\tau=t_{2}-t_{1}$ is the relative delay between photons at the input. The correlation function in our setup is given by $[22,23]$

$$
\begin{aligned}
\Gamma\left(\omega_{1}, \omega_{2}, \tau\right)= & \mid \psi_{1}\left(\omega_{1}\right) \psi_{2}\left(\omega_{2}\right) e^{-i\left(\omega_{1} t_{1}+\omega_{2} t_{2}\right)} \\
& +\left.\psi_{1}\left(\omega_{2}\right) \psi_{2}\left(\omega_{1}\right) e^{-i\left(\omega_{2} t_{1}+\omega_{1} t_{2}\right)}\right|^{2},
\end{aligned}
$$

where $\psi_{1(2)}(\omega)$ is the spectral wave function of the first (second) photon. The spectral correlation function depends on $t_{1}$ and $t_{2}$ only through the relative delay $\tau$ and exhibits interference fringes as a function of $\left(\omega_{1}-\omega_{2}\right)$, with fringe

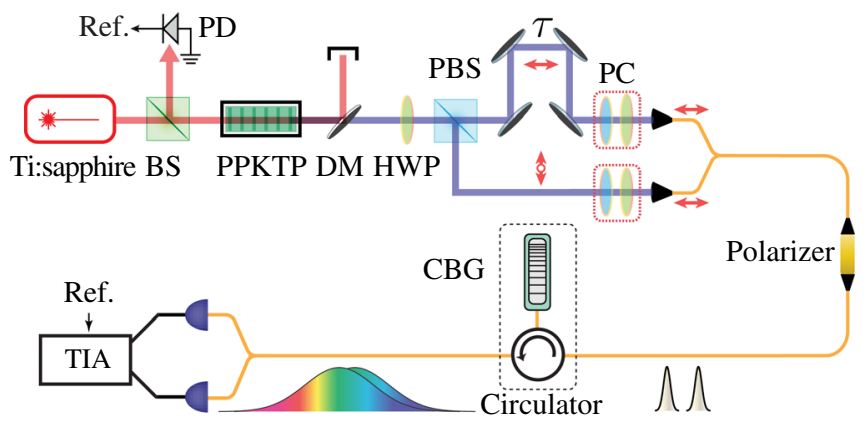

FIG. 1. Schematic of the experimental setup to observe frequency-resolved two-photon interference. Orthogonally polarized photon pairs are generated using Type-II SPDC in a PPKTP crystal, separated using a polarization beam splitter (PBS), delayed and recombined using a beam splitter (BS) after polarization (red arrows) rotation in one of the arms. A chirped Bragg grating $(\mathrm{CBG})$ with a spectral bandwidth more than that of the generated photon pairs, and a time-interval analyzer (TIA) implement a time-of-flight spectrometer (see the Supplemental Material [30]). HWP: half-wave plate (to generate entangled photon pairs), DM: dichroic mirror, PC: polarization controller, PD: photo-diode. separation $2 \pi / \tau$ [28]. Furthermore, because the photons are in a single spatial mode, the unitary transformation describing our optical network adds an overall phase to the photonic wave functions and, therefore, does not contribute to the interference.

In our experiment, we generate photon pairs using spontaneous parametric down-conversion (SPDC) (Fig. 1). A periodically poled potassium titanyl phosphate (PPKTP) crystal (30 mm length) is pumped using a pulsed $(\approx 1.6 \mathrm{ps})$ Ti:sapphire laser $(\approx 775.5 \mathrm{~nm}, 50 \mathrm{~mW})$ which generates orthogonally polarized, spectrally degenerate photon pairs at telecom wavelengths via a Type-II collinear SPDC. We separate the two orthogonally polarized photons using a polarization beam splitter (PBS) and introduce a relative delay $(\tau)$ between them. We rotate the polarization in one of the arms such that the two photons are identically polarized and collect them into a single fiber using a beam splitter. We then use a chirped fiber Bragg grating (CBG), two superconducting nanowire detectors (SNSPDs), and a time-interval analyzer (TIA) to measure the spectral correlations between photons. This setup realizes a time-offlight spectrometer where the arrival time of dispersed photons is used to infer their frequency spectrum [22,23,35-37]. Specifically, the frequency $\omega_{i}$ of a photon detected at the detector $i$ is related to the time-of-arrival $t_{i}^{d}$ at the detector as $\left(\omega_{i}-\omega_{0}\right)=\left(t_{i}^{d}-t_{0 i}^{d}\right) / \phi^{\prime \prime}$. Here, $\omega_{0}$ is the peak frequency of the photonic spectral wave packet and $t_{0 i}^{d}$ is the peak arrival time of the photonic temporal wave packet at the detector $i$. $\phi^{\prime \prime} \simeq 3196 \mathrm{ps}^{2}$ is the group delay dispersion (GDD) of the CBG. In our measurements, we set the central frequency $\omega_{0}$ (corresponding to the time $t_{0}^{d}$ ) to be zero such that $\omega_{i}$ is actually the detuning from the central frequency. The spectral resolution $(\delta \omega)$ of our spectrometer is limited by the timing jitter $(\approx 100 \mathrm{ps})$ of the nanowire detectors and is $\approx 5 \mathrm{GHz}$. Furthermore, the finite delay between the input photons contributes to the timing uncertainty in $t_{0}^{d}$ and marginally lowers the spectral resolution of our spectrometer for input delay values approaching the inherent timing jitter of the detectors.

Figures 2(a)-2(d) show quantum interference fringes in the measured spectral correlations $\left[\Gamma\left(\omega_{1}, \omega_{2}\right)\right.$, twofold coincidences] for different delays between the two photons. The interference fringes can be seen more clearly by plotting the number of coincidences as a function of the frequency separation $\omega_{2}-\omega_{1}$ [Figs. 2(i)-2(1)]. As expected, the fringe separation decreases as $1 / \tau$ [Fig. 2(m)]. Moreover, we see that the visibility (see the Supplemental Material [30]) of the interference decreases with increasing delay [Fig. 2(n)], disappearing completely for $\tau \gtrsim 150 \mathrm{ps}$ [Fig. 2(d)]. This is because of the residual distinguishability following spectrally resolved detection for time delays that approach the inverse of the spectral resolution $\delta \omega$. The interference visibility could, in principle, be restored by increasing the spectral resolution of the detector so that the condition $\delta \omega \gg 1 / \tau$ is satisfied $[20,27,28]$. Our experimental results 


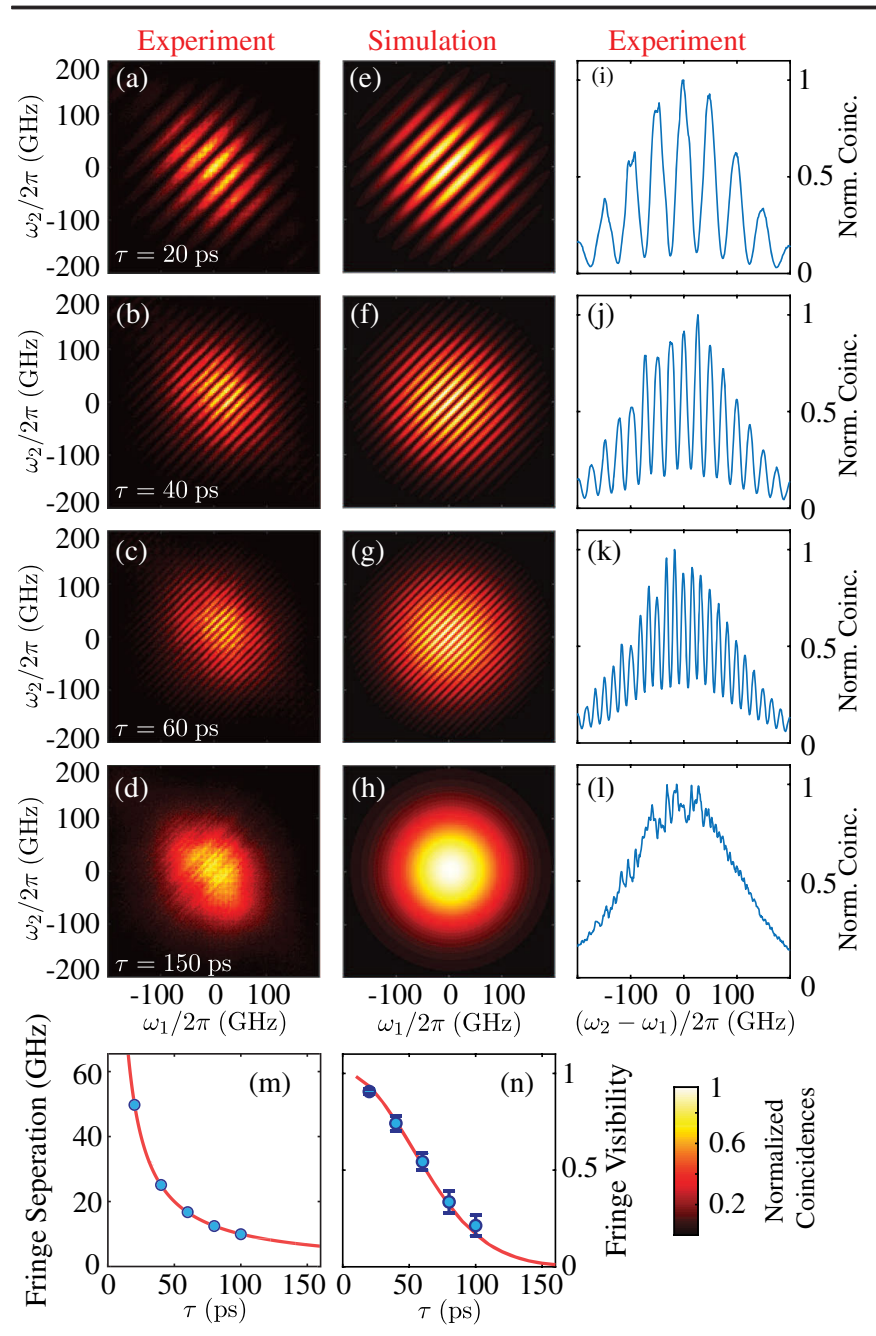

FIG. 2. (a)-(d) Measured and (e)-(h) simulated spectral correlations $\left[\Gamma\left(\omega_{1}, \omega_{2}, \tau\right)\right]$ between two photons with varying relative delay $\tau$ at the input. (i)-(l) Measured coincidence counts (maximum normalized to unity) as a function of frequency separation $\left(\omega_{2}-\omega_{1}\right)$. Measured (blue dots) and simulated (red line) (m) fringe separation, and (n) visibility as function of the delay $\tau$. The delay between the photons was calibrated using HOM interference.

agree well with the simulation results [Figs. 2(e)-2(h)]. We note that similar interference in spectral correlations has been observed in Refs. [22,23] using the two spatial modes of a HOM interference setup. By contrast, in our setup, the two delayed photons are in a single spatial mode. Furthermore, the large GDD of our CBG allows us to observe interference between photons that are separated by delays as long as $100 \mathrm{ps}$, which is more than 50 times the single-photon temporal pulse widths (estimated to be $\approx 1.55 \mathrm{ps}$ ).

Next we discuss the experimental setup and our observation of three-photon interference using frequencyresolved detection. We pump the PPKTP crystal at higher power $(400 \mathrm{~mW})$ to ensure a higher probability of generating two pairs of photons (see the Supplemental Material [30]).

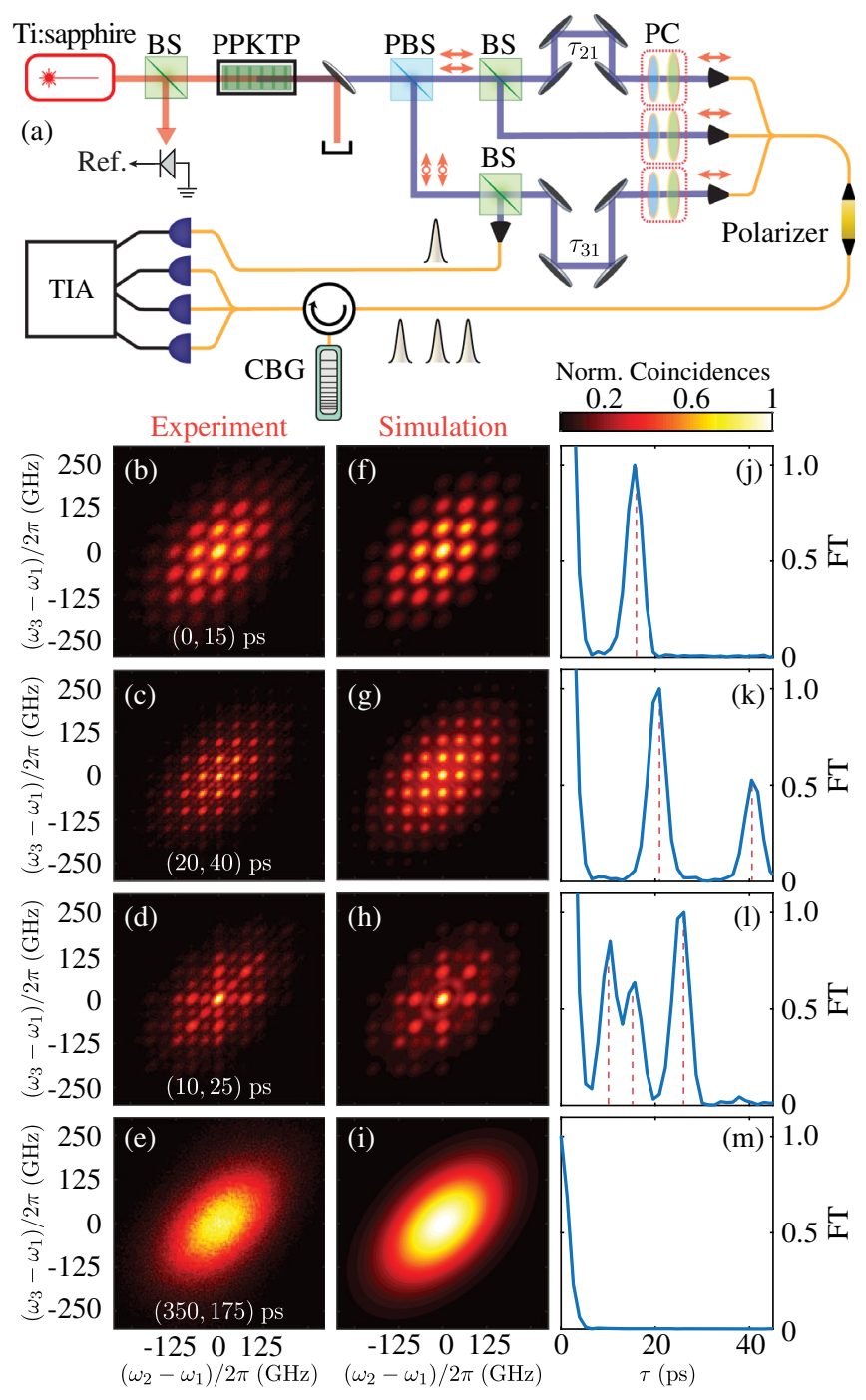

FIG. 3. (a) Schematic of the setup to observe frequency-resolved three-photon interference. The PPKTP crystal is strongly pumped to efficiently generate two pairs of photons which are then probabilistically separated using a PBS and two nonpolarizing beam splitters. (b)-(e) Measured and (f)-(i) simulated spectral correlations between three photons (heralded by the fourth photon) with relative delays $\left(\tau_{21}, \tau_{31}\right) \approx(0,15) ;(20,40) ;(10,25) ;(350,175)$ ps. (j)(m) Fourier transform (FT) of the three-photon correlation function [in (b)-(e)], integrated over $\omega_{3}$. The peaks (highlighted by red dashes) indicate beat notes associated with multiple pairwise interferences.

We use a PBS and two nonpolarizing beam splitters to probabilistically split the four photons into four spatial modes [see Fig. 3(a)]. We then introduce relative delays between the photons using two-photon interference measurements as a calibration tool, and combine three of the four spatial modes into a single fiber using a tritter $(3 \times 3$ beam splitter). As before, the three photons are then dispersed using the $\mathrm{CBG}$, separated using a tritter and their spectral correlations are measured using three detectors connected to the TIA. The fourth photon is used to trigger the TIA. 
Figures 3(b)-3(e) show the measured spectral correlations between the three photons (using fourfold coincidence detections) as a function of the frequency detunings measured at the second and the third detectors, relative to that of the first detector, that is, $\left(\omega_{2}-\omega_{1}\right)$ and $\left(\omega_{3}-\omega_{1}\right)$. We analyze three different delay scenarios $(1)\left(\tau_{21}, \tau_{31}\right) \approx(0,15) \mathrm{ps}$, (2) $(20,40) \mathrm{ps}$, and $(3)(10,25) \mathrm{ps}$, where $\left(\tau_{21}, \tau_{31}\right)$ are the delays of the second and the third photon, respectively, relative to the first photon. We observe that the interference landscape changes significantly with the relative delays between photons. For delays symmetric under exchange of two of the photons $\left(\tau_{21}, \tau_{31}\right) \approx(0,15)$ and $(20,40) \mathrm{ps}$, the interference fringes are periodic along both axes. By contrast, in the case of asymmetric delays $(10,25) \mathrm{ps}$, the constructive correlations are more prominent along the cross sections $\omega_{2}=\omega_{1}$ (vertical), $\omega_{3}=\omega_{1}$ (horizontal), and $\omega_{2}=\omega_{3}$ (diagonal). However, irrespective of the delays between the photons, we always observe a constructive interference for zero frequency detuning, that is, when $\omega_{2}-\omega_{1}=0=$ $\omega_{3}-\omega_{1}$ [see Eq. (1)]. We also analyze the scenario when the spectral resolution of our setup is not high enough to erase the temporal distinguishability of photons [Fig. 3(e)], and, as expected, we do not observe any interference.

We see that the interference patterns shown in Figs. 3(b)-3(e) are symmetric under any permutation of the frequency detunings, for instance, $\omega_{2} \leftrightarrow \omega_{3}$ or $\omega_{3} \leftrightarrow \omega_{1}$, etc. This permutation symmetry is simply a manifestation of the symmetry of our optical network [20]. We again emphasize that the interfering photons propagate in a single fiber. The tritter, together with the three detectors, at the output simply emulates a number-resolving detector and does not contribute to the interference. Moreover, the interference landscape is also symmetric under reflections $\omega_{i} \leftrightarrow-\omega_{i}$, for all $i$, where $i=1,2,3$ is the detector number, because of the symmetric frequency spectra of the input photons [20].

The measured three-photon interference is dictated by the 3 ! three-photon detection amplitudes associated with the possible ways in which the three photons can trigger the three detectors. However, it is instructive to integrate the threephoton correlation function over one of the frequencies (here $\omega_{3}$ ) and analyze the reduced interference as a function of the relative frequency detunings at the other two detectors $\left(\omega_{2}-\omega_{1}\right)$ (see the Supplemental Material [30]). Fourier analysis of this $1 \mathrm{D}$ plot then reveals the beat notes corresponding to the multiple pairwise interference terms between the three photons [Figs. 3(j)-3(m)]. When the input delay values are configured to be $\left(\tau_{21}, \tau_{31}\right) \approx(0,15) \mathrm{ps}$, there is only one possible delay combination between any two photon pairs and the corresponding Fourier transform shows a single peak (highlighted by the dashed red line) at $15.7 \mathrm{ps}$. For $(20,40) \mathrm{ps}$, there are two possible combinations and accordingly we observe two peaks in the Fourier transform, at $20.9 \mathrm{ps}$ and $40.4 \mathrm{ps}$. For $(10,25) \mathrm{ps}$, there are three possible combinations and, as expected, we see three beat notes in the Fourier transform, at $10.4 \mathrm{ps}, 15.7 \mathrm{ps}$, and $26.1 \mathrm{ps}$. The peak delay values agree well with the expected values to within $1.3 \mathrm{ps}$, the temporal resolution of the Fourier transform.

We note that the beam splitters used in our setup to separate photon pairs of the same polarization are not deterministic and lead to possibilities where two photons always arrive with zero delay (see the Supplemental Material [30]). However, these possibilities do not add any new beat notes to the interference pattern, and could easily be removed using two PPKTP crystals to generate two photon pairs. Nevertheless, our experimental observations match very well with our simulations. We achieve a fidelity of $\approx 0.95$ for each of the three scenarios presented in Figs. 3(b)-3(d). The small loss in observed fidelities is because of the small ellipticity in the joint spectral intensity of the photons (see the Supplemental Material [30]).

Finally, we demonstrate frequency-resolved interference of two photons which are entangled in their arrival times. In particular, we consider time-bin entangled states of the form $|\Psi\rangle=|2\rangle_{e}|0\rangle_{l}-e^{-i \varphi}|0\rangle_{e}|2\rangle_{l}$, where both photons at the input are in the "early" time bin (at time $t_{1}$ ) or in the "late" time bin (at $\left.t_{2}\right)$, and $\varphi$ is the phase associated with the delay between the photons. The spectral correlation function at the output of our optical network (Fig. 1) is then given as

$$
\begin{aligned}
\Gamma\left(\omega_{1}, \omega_{2}, \tau\right)= & \mid \psi_{1}\left(\omega_{1}\right) \psi_{2}\left(\omega_{2}\right) e^{-i\left(\omega_{1}+\omega_{2}\right) t_{1}} \\
& +\left.\psi_{1}\left(\omega_{2}\right) \psi_{2}\left(\omega_{1}\right) e^{-i\left(\omega_{1}+\omega_{2}\right) t_{2}}\right|^{2} .
\end{aligned}
$$

The correlation function now exhibits interference fringes as a function of the two-photon phase $\varphi=\left(\omega_{1}+\omega_{2}\right) \tau$, where $\tau=t_{2}-t_{1}$ is the relative delay between the photons. This interference is similar to the time-reversed HOM interference where the two photons are path entangled, that is, they arrive together at either port of the beam splitter [29]. The two photons can then exit the beam splitter in the same port or in different ports, depending on the phase $\varphi$.

To generate time-bin entangled photon pairs we add a half-wave plate, set at an angle of $22.5^{\circ}$, before the PBS in the setup of Fig. 1. The HWP acts as a 50:50 BS in the polarization domain and, when the two-photon spectral wave function is symmetric, it leads to a polarization entangled two-photon state of the form $|\Psi\rangle=|2\rangle_{H}|0\rangle_{V}-$ $|0\rangle_{H}|2\rangle_{V}[33,36,38]$. As before, we use a PBS to introduce a relative delay between the two orthogonal polarization modes and achieve the time-bin entangled state $|\Psi\rangle=$ $|2\rangle_{e}|0\rangle_{l}-e^{-i \varphi}|0\rangle_{e}|2\rangle_{l}$. The phase $\varphi$ was actively stabilized using a continuous-wave telecom laser with tunable wavelength (see the Supplemental Material [30]). We also used a bandpass filter (with $75 \mathrm{GHz}$ bandwidth) to ensure that the two-photon spectral amplitude is symmetric, and verify the entanglement using polarization- and time-resolved coincidence measurements (see the Supplemental Material [30]).

Figures 4(a) and 4(b) show the measured and simulated spectral correlations for the time-bin entangled two-photon state where we set $t_{2}-t_{1}=40 \mathrm{ps}$, much longer than the 


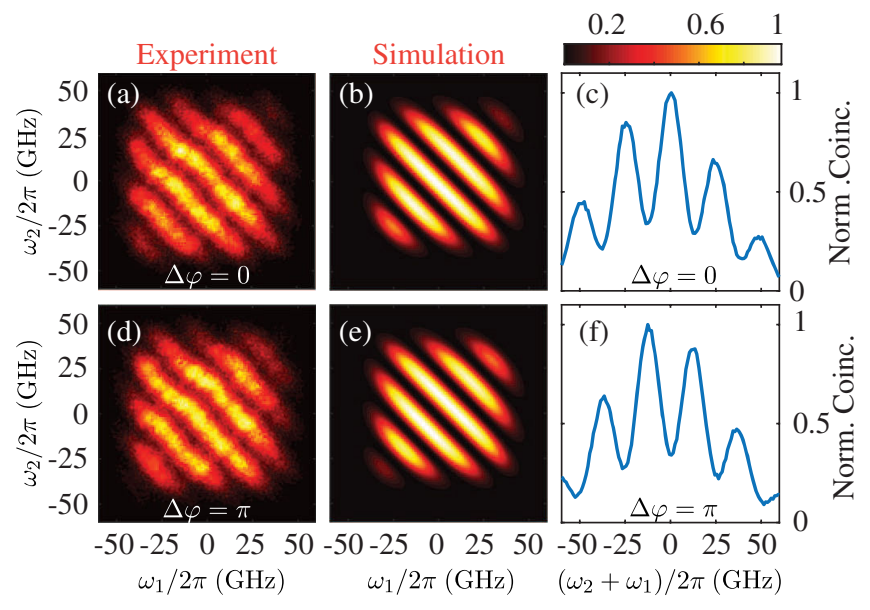

FIG. 4. Measured and simulated spectral correlations for timebin entangled photon pairs, with the phase factor (a)-(b) $\Delta \varphi=0$ and, (d) - (e) $\Delta \varphi=\pi$. (c), (f) Measured coincidences as a function of $\left(\omega_{1}+\omega_{2}\right)$.

single-photon pulsewidths (estimated to be $\approx 5.4 \mathrm{ps}$ following the bandpass filter). We observe interference fringes in the correlations as a function of the two-photon phase $\left(\omega_{1}+\omega_{2}\right) \tau$ [Fig. 4(c)]. This contrasts with the interference for a separable state of two-photons with a delay (Fig. 2), where interference pattern is rotated by $90^{\circ}$ because of its dependence on $\left(\omega_{1}-\omega_{2}\right) \tau$. Furthermore, as in the timereversed HOM interference, the interference observed here is sensitive to small changes in the two-photon phase $\varphi$. For example, by introducing an additional small delay $\Delta \tau$ (few fs) such that $\Delta \varphi=\left(\omega_{1}+\omega_{2}\right) \Delta \tau=\pi$, we observe the complimentary interference where the peaks are replaced by troughs and vice versa [Figs. 4(d)-4(f)]. We note that the marginal decrease in the observed visibility for interference of time-bin entangled photons compared to the unentangled photons (Fig. 2) is because of the imperfections in the entangled state preparation and the sensitivity to residual path length fluctuations in the interferometer (see the Supplemental Material [30]).

In summary, we have demonstrated frequency-resolved interference of three photons that are separated in time using a single dispersive element. Using a larger number of photons and a time-varying dispersion element, such as a phase modulator, our setup could realize temporal boson sampling in a single spatial mode with easily reconfigurable unitary transformation and explore phase transitions in the complexity of sampling [28,30,39]. Our scheme can also be used to implement scalable multiboson correlation sampling where the photonic correlations are sampled over spatial as well as temporal or spectral modes at the input or ouput of a random linear optical network with multiple spatial modes [24,27]. Finally, these experimental results may pave the way to new techniques for the experimental characterization of optical networks and their input photonic states with potential application in quantum information processing and metrology $[20,40,41]$.
This research was supported by the Air Force Office of Scientific Research Multidisciplinary University Research Initiative (AFOSR-MURI Grant No. FA9550-16-1-0323) and the Physics Frontier Center at the Joint Quantum Institute. A. D. and A. V. G. acknowledge funding by ARL CDQI, ARO MURI, NSF PFC at JQI, AFOSR, NSF PFCQC program, DOE ASCR Quantum Testbed Pathfinder program (Award No. DE-SC0019040), and DOE BES QIS program (Award No. DE-SC0019449). V. T. was partially supported by the Office of Naval Research Global (N62909-18-1-2153). We thank Hana Warner and Jessica Christian for help with the experimental setup, and Qudsia Quraishi for the nanowire detectors.

*mittals@umd.edu

[1] E. Knill, R. Laflamme, and G. J. Milburn, Nature (London) 409, 46 (2001).

[2] J. Carolan, C. Harrold, C. Sparrow, E. Martín-López, N. J. Russell, J. W. Silverstone, P. J. Shadbolt, N. Matsuda, M. Oguma, M. Itoh et al., Science 349, 711 (2015).

[3] T. D. Ladd, F. Jelezko, R. Laflamme, Y. Nakamura, C. Monroe, and J. L. OBrien, Nature (London) 464, 45 (2010).

[4] J.-W. Pan, Z.-B. Chen, C.-Y. Lu, H. Weinfurter, A. Zeilinger, and M. Żukowski, Rev. Mod. Phys. 84, 777 (2012).

[5] D. Bouwmeester, J.-W. Pan, K. Mattle, M. Eibl, H. Weinfurter, and A. Zeilinger, Nature (London) 390, 575 (1997).

[6] K. Mattle, H. Weinfurter, P. G. Kwiat, and A. Zeilinger, Phys. Rev. Lett. 76, 4656 (1996).

[7] H.-K. Lo, M. Curty, and K. Tamaki, Nat. Photonics 8, 595 (2014).

[8] J. P. Dowling, Contemp. Phys. 49, 125 (2008).

[9] G. B. Lemos, V. Borish, G. D. Cole, S. Ramelow, R. Lapkiewicz, and A. Zeilinger, Nature (London) 512, 409 (2014).

[10] K. R. Motes, A. Gilchrist, J. P. Dowling, and P. P. Rohde, Phys. Rev. Lett. 113, 120501 (2014).

[11] S. Aaronson and A. Arkhipov, Theory Comput. 9, 143 (2013).

[12] N. Spagnolo, C. Vitelli, M. Bentivegna, D. J. Brod, A. Crespi, F. Flamini, S. Giacomini, G. Milani, R. Ramponi, P. Mataloni et al., Nat. Photonics 8, 615 (2014).

[13] C. K. Hong, Z. Y. Ou, and L. Mandel, Phys. Rev. Lett. 59, 2044 (1987).

[14] Y. H. Shih and C. O. Alley, Phys. Rev. Lett. 61, 2921 (1988).

[15] T. Legero, T. Wilk, A. Kuhn, and G. Rempe, Appl. Phys. B 77, 797 (2003).

[16] T. Legero, T. Wilk, M. Hennrich, G. Rempe, and A. Kuhn, Phys. Rev. Lett. 93, 070503 (2004).

[17] T.-M. Zhao, H. Zhang, J. Yang, Z.-R. Sang, X. Jiang, X.-H. Bao, and J.-W. Pan, Phys. Rev. Lett. 112, 103602 (2014).

[18] G. Vittorini, D. Hucul, I. V. Inlek, C. Crocker, and C. Monroe, Phys. Rev. A 90, 040302(R) (2014).

[19] V. Tamma and S. Laibacher, Phys. Rev. Lett. 114, 243601 (2015).

[20] S. Laibacher and V. Tamma, Phys. Rev. A 98, 053829 (2018). 
[21] X.-J. Wang, B. Jing, P.-F. Sun, C.-W. Yang, Y. Yu, V. Tamma, X.-H. Bao, and J.-W. Pan, Phys. Rev. Lett. 121, 080501 (2018).

[22] T. Gerrits, F. Marsili, V. B. Verma, L. K. Shalm, M. Shaw, R. P. Mirin, and S. W. Nam, Phys. Rev. A 91, 013830 (2015).

[23] R.-B. Jin, T. Gerrits, M. Fujiwara, R. Wakabayashi, T. Yamashita, S. Miki, H. Terai, R. Shimizu, M. Takeoka, and M. Sasaki, Opt. Express 23, 28836 (2015).

[24] S. Laibacher and V. Tamma, Phys. Rev. Lett. 115, 243605 (2015).

[25] V. Tamma and S. Laibacher, Quantum Inf. Process. 15, 1241 (2016).

[26] V. Tamma and S. Laibacher, J. Mod. Opt. 63, 41 (2016).

[27] S. Laibacher and V. Tamma, arXiv:1801.03832.

[28] M. Pant and D. Englund, Phys. Rev. A 93, 043803 (2016).

[29] J. Chen, K. F. Lee, and P. Kumar, Phys. Rev. A 76, 031804 (R) (2007).

[30] See Supplemental Material at http://link.aps.org/ supplemental/10.1103/PhysRevLett.123.123603 for details on the experimental setup, which includes Refs. [28,31-34].

[31] V. Giovannetti, L. Maccone, J. H. Shapiro, and F. N. C. Wong, Phys. Rev. A 66, 043813 (2002).
[32] W. P. Grice and I. A. Walmsley, Phys. Rev. A 56, 1627 (1997).

[33] S. Slussarenko, M. M. Weston, H. M. Chrzanowski, L. K. Shalm, V. B. Verma, S. W. Nam, and G. J. Pryde, Nat. Photonics 11, 700 (2017).

[34] S. Aaronson and A. Arkhipov, in Proceedings of the 43rd annual ACM symposium on Theory of computing (STOC '11) (ACM, New York, 2011), pp. 333-342.

[35] M. Avenhaus, A. Eckstein, P. J. Mosley, and C. Silberhorn, Opt. Lett. 34, 2873 (2009).

[36] S. Mittal, V. V. Orre, A. Restelli, R. Salem, E. A. Goldschmidt, and M. Hafezi, Phys. Rev. A 96, 043807 (2017).

[37] A. O. C. Davis, P. M. Saulnier, M. Karpiński, and B. J. Smith, Opt. Express 25, 12804 (2017).

[38] P. S. Kuo, T. Gerrits, V. B. Verma, and S. W. Nam, Opt. Lett. 41, 5074 (2016).

[39] A. Deshpande, B. Fefferman, M. C. Tran, M. Foss-Feig, and A. V. Gorshkov, Phys. Rev. Lett. 121, 030501 (2018).

[40] D. Oren, M. Mutzafi, Y. C. Eldar, and M. Segev, Optica 4, 993 (2017).

[41] K. Wang, S. V. Suchkov, J. G. Titchener, A. Szameit, and A. A. Sukhorukov, Optica 6, 41 (2019). 